\title{
Weak Signed Roman Domination in Digraphs
}

\author{
Lutz Volkmann
}

\begin{abstract}
Let $D$ be a finite and simple digraph with vertex set $V(D)$. A weak signed Roman dominating function (WSRDF) on a digraph $D$ is a function $f: V(D) \rightarrow\{-1,1,2\}$ satisfying the condition that $\sum_{x \in N^{-}[v]} f(x) \geq 1$ for each $v \in V(D)$, where $N^{-}[v]$ consists of $v$ and all vertices of $D$ from which arcs go into $v$. The weight of a WSRDF $f$ is $\sum_{v \in V(D)} f(v)$. The weak signed Roman domination number $\gamma_{w s R}(D)$ of $D$ is the minimum weight of a WSRDF on $D$. In this paper we initiate the study of the weak signed Roman domination number of digraphs, and we present different bounds on $\gamma_{w s R}(D)$. In addition, we determine the weak signed Roman domination number of some classes of digraphs.
\end{abstract}

\section{Terminology and introduction}

In this paper we continue the study of Roman dominating functions in graphs and digraphs. Let $G$ be a simple graph with vertex set $V(G)$, and let $N[v]=N_{G}[v]$ be the closed neighborhood of the vertex $v$.

A signed Roman dominating function on a graph $G$ is defined in [1] as a function $f: V(G) \longrightarrow$ $\{-1,1,2\}$ satisfying the conditions that (i) $f\left(N_{G}[v]\right)=\sum_{x \in N_{G}[v]} f(x) \geq 1$ for every $v \in V(G)$, and (ii) for every vertex $u$ for which $f(u)=-1$ is adjacent to a vertex $v$ for which $f(v)=2$. The weight of a signed Roman dominating function $f$ on a graph $G$ is $\sum_{v \in V(G)} f(v)$. The signed Roman domination number $\gamma_{s R}(G)$ of $G$ is the minimum weight of a signed Roman dominating function on $G$.

A weak signed Roman dominating function on a graph $G$ is defined in [14] as a function $f: V(G) \longrightarrow\{-1,1,2\}$ such that $f\left(N_{G}[v]\right) \geq 1$ for every $v \in V(G)$. The weight of a weak signed Roman dominating function $f$ on a graph $G$ is $\sum_{v \in V(G)} f(v)$. The weak signed Roman domination number $\gamma_{w s R}(G)$ of $G$ is the minimum weight of a weak signed Roman dominating function on $G$. Clearly, $\gamma_{w s R}(G) \leq \gamma_{s R}(G)$.

Key words and phrases. Digraph, Signed Roman domination number, Weak signed Roman domination number.

2010 Mathematics Subject Classification. 05C20, 05 C69. 
Let now $D$ be a finite and simple digraph with vertex set $V(D)$ and $\operatorname{arcset} A(D)$. The integers $n(D)=|V(D)|$ and $m(D)=|A(D)|$ are the order and the size of the digraph $D$, respectively. We write $d_{D}^{+}(v)=d^{+}(v)$ for the out-degree of a vertex $v$ and $d_{D}^{-}(v)=d^{-}(v)$ for its in-degree. The minimum and maximum in-degree are $\delta^{-}(D)$ and $\Delta^{-}(D)$ and the minimum and maximum out-degree are $\delta^{+}(D)$ and $\Delta^{+}(D)$. The sets $N_{D}^{+}(v)=N^{+}(v)=\{x \mid(v, x) \in A(D)\}$ and $N_{D}^{-}(v)=N^{-}(v)=\{x \mid(x, v) \in A(D)\}$ are called the out-neighborhood and in-neighborhood of the vertex $v$. Likewise, $N_{D}^{+}[v]=N^{+}[v]=N^{+}(v) \cup\{v\}$ and $N_{D}^{-}[v]=N^{-}[v]=N^{-}(v) \cup\{v\}$. If $X \subseteq V(D)$, then $D[X]$ is the subdigraph induced by $X$. For an $\operatorname{arc}(x, y) \in A(D)$, the vertex $y$ is an out-neighbor of $x$ and $x$ is an in-neighbor of $y$, and we also say that $x$ dominates $y$ or $y$ is dominated by $x$. For a real-valued function $f: V(D) \longrightarrow \mathbf{R}$, the weight of $f$ is $w(f)=$ $\sum_{v \in V(D)} f(v)$, and for $S \subseteq V(D)$, we define $w_{f}(S)=\sum_{v \in S} f(v)$, so $w(f)=w_{f}(V(D))$. Consult [5] or [6] for notation and terminology which are not defined here.

We define a set $S \subseteq V(D)$ to be a dominating set of $D$ if for all $v \notin S, v$ is dominated by a vertex $s \in S$. The minimum cardinality of a dominating set in $D$ is the domination number $\gamma(D)$.

In this paper, we continue the study of signed Roman domination in graphs and digraphs (see, for example, $[1,2,3,4,7,8,9,10,11,12,13,14]$ ).

A signed Roman dominating function (abbreviated SRDF) on $D$ is defined in [9] as a function $f: V(D) \longrightarrow\{-1,1,2\}$ such that $f\left(N^{-}[v]\right)=\sum_{x \in N^{-}[v]} f(x) \geq 1$ for every $v \in V(D)$ and every vertex $u$ for which $f(u)=-1$ has an in-neighbor $v$ for which $f(v)=2$. The weight of an SRDF $f$ on a digraph $D$ is $w(f)=\sum_{v \in V(D)} f(v)$. The signed Roman domination number $\gamma_{s R}(D)$ of $D$ is the minimum weight of an SRDF on $D$. A $\gamma_{s R}(D)$-function is a signed Roman dominating function on $D$ of weight $\gamma_{s R}(D)$.

A weak signed Roman dominating function (abbreviated WSRDF) on $D$ is defined as a function $f: V(D) \longrightarrow\{-1,1,2\}$ such that $f\left(N^{-}[v]\right) \geq 1$ for every $v \in V(D)$. The weight of a WSRDF $f$ on a digraph $D$ is $w(f)=\sum_{v \in V(D)} f(v)$. The weak signed Roman domination number $\gamma_{w s R}(D)$ of $D$ is the minimum weight of a WSRDF on $D$. A $\gamma_{w s R}(D)$-function is a weak signed Roman dominating function on $D$ of weight $\gamma_{w s R}(D)$. For a WSRDF $f$ on $D$, let $V_{i}=V_{i}(f)=\{v \in V(D): f(v)=i\}$ for $i=-1,1,2$. A weak signed Roman dominating function $f: V(D) \longrightarrow\{-1,1,2\}$ can be represented by the ordered partition $\left(V_{-1}, V_{1}, V_{2}\right)$ of $V(D)$.

The definitions lead to $\gamma_{w s R}(D) \leq \gamma_{s R}(D)$. Therefore each lower bound of $\gamma_{s w R}(D)$ is also a lower bound of $\gamma_{s R}(D)$.

Our purpose in this work is to initiate the study of the weak signed Roman domination number in digraphs. We present basic properties and sharp bounds for the weak signed Roman domination number of digraphs. In particular, we show that many lower bounds on $\gamma_{s R}(D)$ are also valid for $\gamma_{w s R}(D)$. In addition, we show that the difference $\gamma_{s R}(D)-\gamma_{w s R}(D)$ can be arbitrarily large, and we determine the weak signed Roman domination number of some classes of digraphs. 
The associated digraph $D(G)$ of a graph $G$ is the digraph obtained from $G$ when each edge $e$ of $G$ is replaced by two oppositely oriented arcs with the same ends as $e$. Since $N_{D(G)}^{-}[v]=N_{G}[v]$ for each vertex $v \in V(G)=V(D(G))$, the following useful observation is valid.

Observation 1.1. If $D(G)$ is the associated digraph of a graph $G$, then $\gamma_{s R}(D(G))=\gamma_{s R}(G)$ and $\gamma_{w s R}(D(G))=\gamma_{w s R}(G)$.

Let $K_{n}$ and $D\left(K_{n}\right)$ be the complete graph and complete digraph of order $n$, respectively. In [14], the author determines the weak signed Roman domination number of complete graphs.

Proposition 1.1. ([14]) If $n \geq 1$, then $\gamma_{w s R}\left(K_{n}\right)=1$.

Using Observation 1.1 and Proposition 1.1, we obtain the weak signed Roman domination number of complete digraphs.

Corollary 1.2. If $n \geq 1$, then $\gamma_{w s R}\left(D\left(K_{n}\right)\right)=1$.

\section{Preliminary results and bounds}

In this section we present basic properties and some first bounds on the weak signed Roman domination number of digraphs. The definitions immediately lead to our first proposition.

Proposition 2.1. If $f=\left(V_{-1}, V_{1}, V_{2}\right)$ is a WSRDF on a digraph $D$ of order $n$, then

(a) $\left|V_{-1}\right|+\left|V_{1}\right|+\left|V_{2}\right|=n$.

(b) $\omega(f)=\left|V_{1}\right|+2\left|V_{2}\right|-\left|V_{-1}\right|$.

(c) Every vertex in $V_{-1}$ is dominated by one vertex of $V_{2}$ or two vertices of $V_{1}$.

(d) $V_{1} \cup V_{2}$ is a dominating set of $D$.

Proposition 2.2. If $D$ is a digraph of $\operatorname{order} n$, then

$$
\gamma_{w s R}(D) \geq 2+\Delta^{-}(D)-n \text {. }
$$

Proof. Let $w \in V(D)$ be a vertex of maximum in-degree, and let $f$ be a $\gamma_{w s R}(D)$-function. Then the definitions imply

$$
\begin{aligned}
\gamma_{w s R}(D) & =\sum_{x \in V(D)} f(v)=\sum_{x \in N^{-}[w]} f(x)+\sum_{x \in V(D) \backslash N^{-}[w]} f(x) \\
& \geq 1+\sum_{x \in V(D) \backslash N^{-}[w]} f(x) \geq 1-\left(n-\left(\Delta^{-}(D)+1\right)\right)=2+\Delta^{-}(D)-n,
\end{aligned}
$$

and the proof of the desired lower bound is complete. 
Proposition 2.3. If $D$ is a digraph of order $n$ with minimum in-degree $\delta^{-} \geq 2$, then $\gamma_{w s R}(D) \leq$ $n-2\left\lfloor\delta^{-} / 2\right\rfloor$.

Proof. Let $t=\left\lfloor\delta^{-} / 2\right\rfloor$, and let $A=\left\{v_{1}, v_{2}, \ldots, v_{t}\right\}$ be a set of $t$ vertices of $D$. Define the function $f: V(D) \longrightarrow\{-1,1,2\}$ by $f(x)=-1$ for $x \in A$ and $f(x)=1$ for $x \in V(D) \backslash A$. Then

$$
f\left(N^{-}[w]\right) \geq-t+\left(\delta^{-}+1-t\right)=\delta^{-}+1-2 t=\delta^{-}+1-2\left\lfloor\frac{\delta^{-}}{2}\right\rfloor \geq 1
$$

for each $w \in V(D)$. Therefore $f$ is WSRDF on $D$ of weight $n-2 t$ and thus $\gamma_{w s R}(D) \leq n-2 t$.

The proof of the next proposition is identically with the proof of Proposition 6 in [9] and is therefore omitted.

Proposition 2.4. Assume that $f=\left(V_{-1}, V_{1}, V_{2}\right)$ is a WSRDF on a digraph $D$ of order $n$. If $\Delta^{+}(D)=\Delta^{+}$and $\delta^{+}(D)=\delta^{+}$, then

(a) $\left(2 \Delta^{+}+1\right)\left|V_{2}\right|+\Delta^{+}\left|V_{1}\right| \geq\left(\delta^{+}+2\right)\left|V_{-1}\right|$.

(b) $\left(2 \Delta^{+}+\delta^{+}+3\right)\left|V_{2}\right|+\left(\Delta^{+}+\delta^{+}+2\right)\left|V_{1}\right| \geq\left(\delta^{+}+2\right) n$.

(c) $\left(\Delta^{+}+\delta^{+}+2\right) \omega(f) \geq\left(\delta^{+}-\Delta^{+}+2\right) n+\left(\delta^{+}-\Delta^{+}\right)\left|V_{2}\right|$.

(d) $\omega(f) \geq\left(\delta^{+}-2 \Delta^{+}+1\right) n /\left(2 \Delta^{+}+\delta^{+}+3\right)+\left|V_{2}\right|$.

A digraph $D$ is out-regular or $r$-out-regular if $\delta^{+}(D)=\Delta^{+}(D)=r$, and $D$ is r-regular if $\delta^{+}(D)=\Delta^{+}(D)=\delta^{-}(D)=\Delta^{-}(D)=r$. As an application of Proposition 2.4 (c), we obtain a lower bound on the weak signed Roman domination number for $r$-out-regular digraphs.

Corollary 2.1. If $D$ is an $r$-out-regular digraph of order $n$, then $\gamma_{w s R}(D) \geq n /(r+1)$.

Therefore $\gamma_{s R}(D) \geq n /(r+1)$ for each $r$-out-regular digraph of order $n$ (see [9]). Using Corollary 2.1 and Observation 1.1, we obtain the next known result.

Corollary 2.2. ([1,14]) If $G$ is an $r$-regular graph of order $n$, then $\gamma_{s R}(G) \geq \gamma_{w s R}(G) \geq n /(r+$ $1)$.

If $D$ is not out-regular, then the next lower bound on the weak signed Roman domination number is valid.

Corollary 2.3. Let $D$ be a digraph of order $n$, minimum out-degre $\delta^{+}$and maximum out-degree $\Delta^{+}$. If $\delta^{+}<\Delta^{+}$, then

$$
\gamma_{w s R}(D) \geq\left(\frac{-2 \Delta^{+}+2 \delta^{+}+3}{2 \Delta^{+}+\delta^{+}+3}\right) n
$$


Proof. Multiplying both sides of the inequality in Proposition 2.4 (d) by $\Delta^{+}-\delta^{+}$and adding the resulting inequality to the inequality in Proposition 2.4 (c), we obtain the desired lower bound.

Since $\Delta^{+}(D(G))=\Delta(G)$ and $\delta^{+}(D(G))=\delta(G)$, Corollary 2.3 and Observation 1.1 lead to the next known corollary.

Corollary 2.4. ([1,14]) Let $G$ be a graph of order $n$, minimum degree $\delta$ and maximum degree $\Delta$. If $\delta<\Delta$, then

$$
\gamma_{s R}(G) \geq \gamma_{w s R}(G) \geq\left(\frac{-2 \Delta+2 \delta+3}{2 \Delta+\delta+3}\right) n .
$$

Example 1. Let $p \geq 2$ be an integer, and let $v_{1}, v_{2}, \ldots, v_{p}$ be the vertex set of the complete graph $K_{p}$. Next let $H$ be the graph consisting of $K_{p}$ such that each vertex $v_{i}$ is adjacent to $2 p-1$ leaves for $1 \leq i \leq p$. Now let $D(H)$ be the associated digraph of $H$. Define the function $f: V(D(H)) \longrightarrow$ $\{-1,1,2\}$ by $f\left(v_{i}\right)=2$ for $1 \leq i \leq p$ and $f(x)=-1$ otherwise. Then $f$ is a WSRDF on $D(H)$ of weight

$$
3 p-2 p^{2}=\left(\frac{-2 \Delta^{+}(D(H))+2 \delta^{+}(D(H))+3}{2 \Delta^{+}(D(H))+\delta^{+}(D(H))+3}\right) n(D(H)) .
$$

Therefore Corollary 2.3 shows $\gamma_{w s R}(D(H))=3 p-2 p^{2}$ and thus Corollary 2.3 is sharp.

Since $f$ is also a signed Roman dominating function on $D(H)$, this example demonstrates that the inequality

$$
\gamma_{s R}(D) \geq\left(\frac{-2 \Delta^{+}+2 \delta^{+}+3}{2 \Delta^{+}+\delta^{+}+3}\right) n
$$

which can be found in [9] and which follows from Corollary 2.3, is sharp too.

\section{Special families of digraphs}

In this section, we determine the weak signed Roman domination number for special classes of digraphs. A tournament is a digraph in which for every pair $u, v$ of different vertices, either $(u, v) \in A(D)$ or $(v, u) \in A(D)$, but not both.

The acyclic tournament $A T(n)$ with $n$ vertices has the vertex set $V(A T(n))=\left\{u_{1}, u_{2}, \ldots, u_{n}\right\}$. An arc goes from $u_{i}$ into $u_{j}$ if and only if $i<j$.

Let $n$ be an odd positive integer such $n=2 r+1$ with a positive integer $r$. We define the circulant tournament $\mathrm{CT}(n)$ with $n$ vertices as follows. The vertex set of $\mathrm{CT}(n)$ is $V(\mathrm{CT}(n))=$ $\left\{u_{0}, u_{1}, \ldots, u_{n-1}\right\}$. For each $i$, the arcs are going from $u_{i}$ to the vertices $u_{i+1}, u_{i+2}, \ldots, u_{i+r}$, where the indices are taken modulo $n$.

The proof of the next four propositions are similar to the the proofs of Propositions 7, 8, 11 and 12 in [9] and are therefore omitted. 
Proposition 3.1. If $A T(n)$ is an acyclic tournament for $n \geq 3$, then $\gamma_{w s R}(A T(n))=1$.

Proposition 3.2. Let $n=2 r+1$, where $r \geq 1$ is an integer. Then $\gamma_{w s R}(\mathrm{CT}(n))=3$.

Let $C_{n}=v_{1} v_{2} \ldots v_{n} v_{1}$ be a cycle of order $n$. If we replace each edge $v_{i} v_{i+1}$ by an arc $\left(v_{i}, v_{i+1}\right)$ for $1 \leq i \leq n$, where the indices are taken modulo $n$, then we obtain an oriented cycle $C_{n}^{o}$ of order $n$. Let $P_{n}=v_{1} v_{2} \ldots v_{n}$ be a path of order $n$. If we replace each edge $v_{i} v_{i+1}$ by an arc $\left(v_{i}, v_{i+1}\right)$ for $1 \leq i \leq n-1$, then we obtain an oriented path $P_{n}^{o}$ of order $n$.

Proposition 3.3. Let $C_{n}^{o}$ be an oriented cycle of order $n \geq 2$. Then $\gamma_{w s R}\left(C_{n}^{o}\right)=n / 2$ when $n$ is even and $\gamma_{w s R}\left(C_{n}^{o}\right)=(n+3) / 2$ when $n$ is odd.

Proposition 3.4. Let $P_{n}^{o}$ be an oriented path of order $n$. Then $\gamma_{w s R}\left(P_{n}^{o}\right)=n / 2$ when $n$ is even and $\gamma_{w s R}\left(P_{n}^{o}\right)=(n+1) / 2$ when $n$ is odd.

Let $D\left(K_{p, q}\right)$ be the complete bipartite digraph with partite sets $X$ and $Y$, where $|X|=p$ and $|Y|=q$.

Proposition 3.5. If $q \geq 1$ is an integer, then $\gamma_{w s R}\left(D\left(K_{1, q}\right)\right)=1$ unless $q=2$, in which case $\gamma_{w s R}\left(D\left(K_{1,2}\right)\right)=2$.

Proof. Let $X=\{x\}$ and let $Y=\left\{y_{1}, y_{2}, \ldots, y_{q}\right\}$. According to Proposition 2.2, we have

$$
\gamma_{w s R}\left(D\left(K_{1, q}\right)\right) \geq 2+\Delta^{-}\left(D\left(K_{1, q}\right)\right)-(q+1)=1
$$

If $q=2 t+1$ is odd for an integer $t \geq 0$, then define $f(x)=2, f\left(y_{i}\right)=-1$ for $1 \leq i \leq t+1$ and $f\left(y_{i}\right)=1$ for $t+2 \leq i \leq 2 t+1$. Then $f$ is a WSRDF on $D\left(K_{1, q}\right)$ of weight 1 and therefore $\gamma_{w s R}\left(D\left(K_{1, q}\right)\right)=1$ when $q$ is odd.

If $q=2 t$ is even for an integer $t \geq 1$, then it is easy to see that $\gamma_{w s R}\left(D\left(K_{1,2}\right)\right)=2$. In the case $t \geq 2$ define $f(x)=2, f\left(y_{i}\right)=-1$ for $1 \leq i \leq t+1$ and $f\left(y_{i}\right)=1$ for $t+2 \leq i \leq 2 t-1$ and $f\left(y_{2 t}\right)=2$. Then $f$ is a WSRDF on $D\left(K_{1, q}\right)$ of weight 1 and therefore $\gamma_{w s R}\left(D\left(K_{1, q}\right)\right)=1$ when $q \geq 4$ is even.

Proposition 3.6. Let $2 \leq p \leq q$ be integers. Then $\gamma_{w s R}\left(D\left(K_{2, q}\right)\right)=2, \gamma_{w s R}\left(D\left(K_{3, q}\right)\right)=3$ and $\gamma_{w s R}\left(D\left(K_{p, q}\right)\right)=4$ for $4 \leq p$.

Proof. Let $X=\left\{x_{1}, x_{2}, \ldots, x_{p}\right\}, Y=\left\{y_{1}, y_{2}, \ldots, y_{q}\right\}$, and let $f$ be a $\gamma_{w s R}\left(D\left(K_{p, q}\right)\right)$-function. Assume first that $f\left(x_{i}\right) \geq 1$ for $1 \leq i \leq p$. Then it follows that

$$
\gamma_{w s R}\left(D\left(K_{p, q}\right)\right)=f\left(N^{-}\left[x_{1}\right]\right)+f\left(X \backslash\left\{x_{1}\right\}\right) \geq 1+p-1=p .
$$


If $f\left(y_{i}\right) \geq 1$ for $1 \leq i \leq q$, then we obtain analogously $\gamma_{w s R}\left(D\left(K_{p, q}\right)\right) \geq q \geq p$. Now assume, without loss of generality, that $f\left(x_{1}\right)=f\left(y_{1}\right)=-1$. Then we deduce

$$
\gamma_{w s R}\left(D\left(K_{p, q}\right)\right)=f\left(N^{-}\left[x_{1}\right]\right)+f\left(N^{-}\left[y_{1}\right]\right)-f\left(x_{1}\right)-f\left(y_{1}\right) \geq 1+1+1+1=4 .
$$

Therefore we see that $\gamma_{w s R}\left(D\left(K_{2, q}\right)\right) \geq 2, \gamma_{w s R}\left(D\left(K_{3, q}\right)\right) \geq 3$ and $\gamma_{w s R}\left(D\left(K_{p, q}\right)\right) \geq 4$ for $p \geq 4$.

Conversely, let $p=2$ or $p=3$ and let $q$ be even. Assign to $x \in X$ the weight 1 , to $q / 2$ vertices the weight 1 and to the remaining $q / 2$ vertices of $Y$ the weight -1 . This produces a WSRDF on $D\left(K_{i, q}\right)$ of weight $i$ and therefore $\gamma_{w s R}\left(D\left(K_{i, q}\right)\right)=i$ for $i=2,3$ when $q$ is even. Now let $p=2$ or $p=3$ and let $q=2 s+1$ be odd. Assign to $x \in X$ the weight 1 , to $s+1$ vertices the weight -1 , to one vertex the weight 2 and to the remaining $s-1$ vertices of $Y$ the weight 1 . This produces a WSRDF on $D\left(K_{i, q}\right)$ of weight $i$ and therefore $\gamma_{w s R}\left(D\left(K_{i, q}\right)\right)=i$ for $i=2,3$ when $q$ is odd.

Finally, let $p \geq 4$. Assume that $p$ and $q$ are even. Assign to $(p+2) / 2$ vertices the weight 1 and to the remaining $(p-2) / 2$ vertices of $X$ the weight -1 . In addition, assign to $(q+2) / 2$ vertices the weight 1 and to the remaining $(q-2) / 2$ vertices of $Y$ the weight -1 . This produces a WSRDF on $D\left(K_{p, q}\right)$ of weight 4 and therefore $\gamma_{w s R}\left(D\left(K_{p, q}\right)\right)=4$ in this case.

Next assume that $p=2 t+1$ and $q=2 s+1$ are odd. Assign to $t$ vertices the weight -1 , to one vertex the weight 2 and to the remaining $t$ vertices of $X$ the weight 1 . In addition assign to $s$ vertices the weight -1 , to one vertex the weight 2 and to the remaining $s$ vertices of $Y$ the weight 1. This produces a WSRDF on $D\left(K_{p, q}\right)$ of weight 4 and therefore $\gamma_{w s R}\left(D\left(K_{p, q}\right)\right)=4$ in this case.

The cases $p$ even and $q$ odd or $p$ odd and $q$ even are analogously, and are therefore omitted.

Let $C_{n}$ be a cycle of length $n \geq 3$. In [1], the authors have shown that $\gamma_{s R}\left(C_{n}\right)=\lceil 2 n / 3\rceil$, and in [14] it was shown that $\gamma_{w s R}\left(C_{n}\right)=\lceil n / 3\rceil$ when $n \equiv 0,1(\bmod 3)$ and $\gamma_{w s R}\left(C_{n}\right)=$ $\lceil n / 3\rceil+1$ when $n \equiv 2(\bmod 3)$. Using Observation 1.1 , we deduce that $\gamma_{s R}\left(D\left(C_{n}\right)\right)=\lceil 2 n / 3\rceil$ and $\gamma_{w s R}\left(D\left(C_{n}\right)\right)=\lceil n / 3\rceil$ when $n \equiv 0,1(\bmod 3)$ and $\gamma_{w s R}\left(D\left(C_{n}\right)\right)=\lceil n / 3\rceil+1$ when $n \equiv 2(\bmod 3)$ for the associated digraph $D\left(C_{n}\right)$. Therefore the difference $\gamma_{s R}(D)-\gamma_{w s R}(D)$ can be arbitrarily large.

If $D$ is 0-regular, then $\gamma_{w s R}(D)=n$. Corollary 2.1 implies $\gamma_{w s R}(D) \geq\lceil n /(r+1)\rceil$ when $D$ is $r$-regular, and it follows from Proposition 3.3 that $\gamma_{w s R}\left(C_{n}^{o}\right)=n / 2$ when $n$ is even, and we have seen above that $\gamma_{w s R}\left(D\left(C_{n}\right)\right)=\lceil n / 3\rceil$ when $n \equiv 0,1(\bmod 3)$. Therefore Corollary 2.1 is tight for $r=0,1,2$. In addition, Corollary 1.2 implies that Corollary 2.1 is tight for $r=n-1$. Next we will show that Corollary 2.1 is tight for $r=n-2$ and $r=n-3$. 
Example 2. Let $H=K_{n_{1}, n_{2}, \ldots, n_{r}}$ be the complete $r$-partite graph with $r \geq 2$ and $n_{1}=n_{2}=$ $\ldots=n_{r}=2$, and let $D(H)$ be its associated digraph. Corollary 2.1 implies $\gamma_{w s R}(D(H)) \geq$ $\lceil n /(n-1)\rceil=2$.

Now let $X_{i}=\left\{x_{i}, y_{i}\right\}$ be the partite sets of $H$ for $1 \leq i \leq r$. Define $f\left(x_{i}\right)=f\left(y_{1}\right)=1$ for $1 \leq i \leq r$ and $f\left(y_{i}\right)=-1$ for $2 \leq i \leq r$. Then $f$ is a WSRDF on $D(H)$ of weight 2 and thus $\gamma_{w s R}(D(H)) \leq 2$. Therefore $\gamma_{w s R}(D(H))=2$.

Example 3. Let $F=K_{n_{1}, n_{2}, \ldots, n_{r}}$ be the complete $r$-partite graph with $r \geq 2$ and the partite sets $X_{1}, X_{2}, \ldots, X_{r}$ such that $\left|X_{1}\right|=|\{a, b, u, v\}|=4$ and $\left|X_{i}\right|=3$ for $2 \leq i \leq r$. Let $H$ consisting of $F$ with the additional edges $a b$ and $u v$, and let $D(H)$ be its associated digraph. Then $D(H)$ is $(n-3)$-regular, and Corollary 2.1 implies $\gamma_{s R}(D(H)) \geq \gamma_{w s R}(D(H)) \geq\lceil n /(n-2)\rceil=2$.

Now let $X_{i}=\left\{x_{i}, y_{i}, z_{i}\right\}$ be the partite sets of $H$ for $2 \leq i \leq r$. Define $f\left(x_{i}\right)=f(a)=$ $f(u)=2$ for $2 \leq i \leq r$ and $f(b)=f(v)=f\left(y_{i}\right)=f\left(z_{i}\right)=-1$ for $2 \leq i \leq r$. Then $f$ is a WSRDF (even an SRDF) on $D(H)$ of weight 2 and thus $\gamma_{w s R}(D(H)) \leq \gamma_{s R}(D(H)) \leq 2$. Therefore $\gamma_{w s R}(D(H))=\gamma_{s R}(D(H))=2$.

\section{Further bounds}

The underlying graph of a digraph $D$ is that graph obtained by replacing each arc $(x, y)$ or symmetric pairs $(x, y),(y, x)$ of arcs by the edge $x y$. A digraph $D$ is connected if its underlying graph is connected.

Let $n \geq 2$ be an integer, and let $K_{1, n-1}$ be a star with center $u$ and the leaves $v_{1}, v_{2}, \ldots, v_{n-1}$. Now let $A_{n}$ be an orientation of $K_{1, n-1}$ such that $\Delta^{+}\left(A_{n}\right)=n-1$. If we add one arc $\left(v_{i}, u\right)$ to the digraph $A_{n}$ for an index $i \in\{1,2, \ldots, n-1\}$, then we denote the resulting digraph by $F_{n}$.

Theorem 4.1. Let $D$ be a connected digraph of order $n \geq 2$. Then

$$
\gamma_{s R}(D) \geq \gamma_{w s R}(D) \geq 3-n
$$

In addition, we have $\gamma_{s R}(D)=3-n$ or $\gamma_{w s R}(D)=3-n$ if and only if $D=A_{n}$ or $D=F_{n}$.

Proof. Since $D$ is connected, we observe that $\Delta^{-}(D) \geq 1$, and therefore it follows from Proposition 2.2 that

$$
\gamma_{s R}(D) \geq \gamma_{w s R}(D) \geq 2+\Delta^{-}(D)-n \geq 3-n .
$$

If $D=A_{n}$ or $D=F_{n}$, then define the function $g: V(D) \longrightarrow\{-1,1,2\}$ by $g(u)=2$ and $g\left(v_{i}\right)=-1$ for $1 \leq i \leq n-1$. Then $g$ is a WSRDF and an SRDF on $D$ of weight $3-n$ and thus $\gamma_{w s R}(D) \leq \gamma_{s R}(D) \leq 3-n$. This implies that $\gamma_{s R}(D)=\gamma_{w s R}(D)=3-n$ if $D=A_{n}$ or $D=F_{n}$. 
Conversely, assume that $\gamma_{w s R}(D)=3-n$. If $\Delta^{-}(D) \geq 2$, then Proposition 2.2 implies the contradiction

$$
\gamma_{w s R}(D) \geq 2+\Delta^{-}(D)-n \geq 4-n>3-n .
$$

Therefore let now $\Delta^{-}(D)=1$, and let $f$ be a $\gamma_{w s R}(D)$-function. The condition $\gamma_{w s R}(D)=3-n$ shows that there exists a vertex $v_{1}$ with $f\left(v_{1}\right)=-1$. Since $\Delta^{-}(D)=1$, the vertex $v_{1}$ has exactly one in-neighbor $u$ with $f(u)=2$. If $V(D) \backslash\left\{u, v_{1}\right\}=\left\{v_{2}, v_{3}, \ldots, v_{n-1}\right\}$, then we deduce from $\gamma_{w s R}(D)=3-n$ that $f\left(v_{i}\right)=-1$ for $2 \leq i \leq n-1$. Thus $v_{2}, v_{3}, \ldots, v_{n-1}$ are out-neighbors of $u$. Because of $\Delta^{-}(D)=1$, we observe that $D$ contains at most one more arc from $v_{i}$ to $u$ for an index $i \in\{1,2, \ldots, n-1\}$. Consequently, $D=A_{n}$ or $D=F_{n}$.

Finally, let $\gamma_{s R}(D)=3-n$. Then (4.1) leads to $\gamma_{w s R}(D)=3-n$, and it follows from above that $D=A_{n}$ or $D=F_{n}$.

Theorem 4.2. [9] Let $D$ be a digraph of order $n$. Then $\gamma_{s R}(D) \leq n$, with equality if and only if $D$ is the disjoint union of isolated vertices and oriented triangles.

Corollary 4.3. Let $D$ be a digraph of order $n$. Then $\gamma_{w s R}(D) \leq n$, with equality if and only if $D$ is the disjoint union of isolated vertices and oriented triangles.

Proof. Theorem 4.2 implies $\gamma_{w s R}(D) \leq \gamma_{s R}(D) \leq n$. If $D$ is the disjoint union of isolated vertices and oriented triangles, then it is easy to see that $\gamma_{w s R}(D)=n$. Conversely, assume that $\gamma_{w s R}(D)=n$. Then it follows that $\gamma_{s R}(D)=n$, and we deduce from Theorem 4.2 that $D$ is the disjoint union of isolated vertices and oriented triangles.

Given a connected digraph $H$, let $L(H)$ denote the sets of all digraphs obtained from $H$ by adding $p_{v} \geq d_{H}^{-}(v)+2$ vertices $x_{1}, x_{2}, \ldots, x_{p_{v}}$ to each $v \in V(H)$ such that $x_{i}$ dominates $v$ for $1 \leq i \leq p_{v}$. Let $\mathcal{H}=\{D \mid D \in L(H)$ for some connected digraph $H\}$.

Theorem 4.4. Let $D$ be a connected digraph of order $n$. Then $\gamma_{w s R}(D) \geq 2 \gamma(D)-n$, with equality if and only if $D$ is an isolated vertex or $D \in \mathcal{H}$.

Proof. If $n=1$, then the result is immediate. Therefore let $n \geq 2$, and let $f=\left(V_{-1}, V_{1}, V_{2}\right)$ be a $\gamma_{w s R}(D)$-function. Then it follows from Proposition 2.1 that

$$
\gamma_{w s R}(D)=\left|V_{1}\right|+2\left|V_{2}\right|-\left|V_{-1}\right|=2\left|V_{1}\right|+3\left|V_{2}\right|-n \geq 2\left|V_{1} \cup V_{2}\right|-n \geq 2 \gamma(D)-n
$$

and the desired bound is proved.

If $D \in L(H)$ for some connected digraph $H$, then define $g: V(D) \longrightarrow\{-1,1,2\}$ by $g(x)=-1$ for $x \in V(H)$ and $g(x)=1$ for $x \in V(D) \backslash V(H)$. Then $g\left(N^{-}[x]\right) \geq d_{H}^{-}(x)+$ $2-\left(d_{H}^{-}(x)+1\right)=1$ for $x \in V(H)$ and $g\left(N^{-}[x]\right)=1$ for $x \in V(D) \backslash V(H)$. Therefore $g$ is 
a WSRDF on $D$ of weight $n(D)-2 n(H)$. In addition, we observe that $\gamma(D)=n(D)-n(H)$ and so

$$
\gamma_{w s R}(D) \leq \omega(g)=n(D)-2 n(H)=2 \gamma(D)-n(D) .
$$

Using (4.2), we obtain $\gamma_{w s R}(D)=2 \gamma(D)-n(D)$.

Conversely, assume that $\gamma_{w s R}(D)=2 \gamma(D)-n(D)$. If $\left|V_{2}\right| \geq 1$, then (4.2) leads to the contradiction

$$
\gamma_{w s R}(D)=2\left|V_{1}\right|+3\left|V_{2}\right|-n>2\left|V_{1} \cup V_{2}\right|-n \geq 2 \gamma(G)-n
$$

Therefore $\left|V_{2}\right|=0$. If $\left|V_{-1}\right|=0$, then $\gamma_{w s R}(D)=\left|V_{1}\right|=n$. Since $D$ is connected and $n \geq 2$, it follows from Corollary 4.3 that $D$ is an oriented triangle $C_{3}^{o}$, and we obtain the contradiction

$$
\gamma_{w s R}\left(C_{3}^{o}\right)=3>1=2 \gamma\left(C_{3}^{o}\right)-3
$$

Thus $\left|V_{-1}\right| \geq 1$. Now, if the induced subdigraph $D\left[V_{1}\right]$ contains an arc $(w, z)$, then, since each vertex of $V_{-1}$ has at least two in-neighbors in $V_{1}$, we observe that $V_{1} \backslash\{z\}$ is a dominating set $D$. Combining this observation with (4.2), we obtain the contradiction

$$
\gamma_{w s R}(D)=2\left|V_{1}\right|-n>2\left|V_{1} \backslash\{z\}\right|-n \geq 2 \gamma(G)-n
$$

Hence $D\left[V_{1}\right]$ consists of isolated vertices. The hypothesis that $D$ is connected therefore shows that $D\left[V_{-1} \mid\right.$ is connected. In addition, since $f\left(N^{-}[v]\right) \geq 1$ for each $v \in V_{-1}$, we see that each such vertex $v$ must have at least $d_{D\left[V_{-1}\right]}^{-}(v)+2$ in-neighbors in $V_{1}$. Altogether, we deduce that $D \in L(H)$, where $D\left[V_{-1}\right]$ is the connected digraph $H$.

Corollary 4.5. Let $D$ be a digraph of order $n$. Then $\gamma_{w s R}(D) \geq 2 \gamma(D)-n$, with equality if and only if the components of $D$ are isolated vertices or elements of $\mathcal{H}$.

A set $S \subseteq V(D)$ is called a 2-packing of the digraph $D$, if $N^{-}[u] \cap N^{-}[v]=\emptyset$ for any two distinct vertices $u, v \in S$. The maximum cardinality of a 2-packing in $D$ is the 2-packing number of $D$, denoted by $\rho=\rho(D)$.

Theorem 4.6. If $D$ is a digraph of order $n$, minimum in-degree $\delta^{-}$and 2-packing number $\rho$, then $\gamma_{w s R}(D) \geq \rho\left(\delta^{-}+2\right)-n$.

Proof. Let $\left\{v_{1}, v_{2}, \ldots, v_{\rho}\right\}$ be a 2-packing of $D$, and let $f$ be a $\gamma_{w s R}(D)$-function. If we define the set $A=\bigcup_{i=1}^{\rho} N^{-}\left[v_{i}\right]$, then since $\left\{v_{1}, v_{2}, \ldots, v_{\rho}\right\}$ is a 2-packing of $D$, we note that

$$
|A|=\sum_{i=1}^{\rho}\left(d^{-}\left(v_{i}\right)+1\right) \geq \rho\left(\delta^{-}+1\right) .
$$


This leads to

$$
\begin{aligned}
\gamma_{w s R}(D) & =\sum_{x \in V(D)} f(x)=\sum_{i=1}^{\rho} f\left(N^{-}\left[v_{i}\right]\right)+\sum_{x \in V(D) \backslash A} f(x) \\
& \geq \rho+\sum_{x \in V(D) \backslash A} f(x) \geq \rho-n+|A| \geq \rho-n+\rho\left(\delta^{-}+1\right)=\rho\left(\delta^{-}+2\right)-n,
\end{aligned}
$$

and the proof is complete.

The next example will demonstrate that the bound in Theorem 4.6 is sharp.

Example 4. Let $F$ be an arbitray digraph of order $t \geq 1$, and for each vertex $v \in V(F)$ add a vertex-disjoint copy of a complete digraph $D\left(K_{s}\right)(s \geq 2)$ and identify the vertex $v$ with one vertex of the added complete digraph. Let $Q$ denote the resulting digraph. Furthermore, let $H_{1}, H_{2}, \ldots, H_{t}$ be the added copies of $D\left(K_{s}\right)$. For $i=1,2, \ldots, t$ let $v_{i}$ be the vertex of $H_{i}$ that is identified with a vertex of $F$. We now construct a WSRDF on $Q$ as follows. For each $i=1,2, \ldots, t$, let $f_{i}$ : $V\left(H_{i}\right) \longrightarrow\{-1,1,2\}$ be a WSRDF on the complete digraph of weight 1 (see Corollary 1.2) such that $f_{i}\left(v_{i}\right) \geq 1$. Now let $f: V(Q) \longrightarrow\{-1,1,2\}$ be the function defined by $f(v)=f_{i}(v)$ for each $v \in V\left(H_{i}\right)$. Then $f$ is a WSRDF of $Q$ of weight $t$ and hence $\gamma_{w s R}(Q) \leq t$. Since $n(Q)=t s$, $\delta^{-}(Q)=s-1$ and $\rho(Q)=t$, Theorem 4.6 implies that $\gamma_{w s R}(Q) \geq \rho(Q)\left(\delta^{-}(Q)+2\right)-n(Q)=t$ and thus $\gamma_{w s R}(Q)=\rho(Q)\left(\delta^{-}(Q)+2\right)-n(Q)=t$.

\section{References}

[1] H.A. Ahangar, M.A. Henning, C. Löwenstein, Y. Zhao and V. Samodivkin, Signed Roman domination in graphs, J. Comb. Optim. 27 (2014), 241-255.

[2] A. Bodaghli, S.M. Sheikholeslami, and L. Volkmann, Twin signed Roman domination numbers in directed graphs, Tamkang J. Math. 47 (2016), 357-371.

[3] N. Dehgardi and L. Volkmann, Signed total Roman $k$-domination in directed graphs, Commun. Comb. Optim. 1 (2016), 165-178.

[4] G. Hao, X. Chen and L. Volkmann, Bounds on the signed Roman $k$-domination number of a digraph, Discuss. Math. Graph Theory 39 (2019), 67-79.

[5] T.W. Haynes, S.T. Hedetniemi, and P.J. Slater, Fundamentals of Domination in Graphs, Marcel Dekker, Inc., New York (1998).

[6] T.W. Haynes, S.T. Hedetniemi, and P.J. Slater, editors, Domination in Graphs, Advanced Topics, Marcel Dekker, Inc., New York (1998). 
[7] M.A. Henning and L. Volkmann, Signed Roman $k$-domination in trees, Discrete Appl. Math. 186 (2015), 98-105.

[8] M.A. Henning and L. Volkmann, Signed Roman $k$-domination in graphs, Graphs Combin. 32 (2016), 175-190.

[9] S.M. Sheikholeslami and L. Volkmann, Signed Roman domination in digraphs, J. Comb. Optim. 30 (2015), 456-467.

[10] L. Volkmann, Signed total Roman domination in graphs, J. Comb. Optim. 32 (2016), 855871.

[11] L. Volkmann, Signed Roman k-domination in digraphs, Graphs Combin. 32 (2016), 12171227.

[12] L. Volkmann, Signed total Roman domination in digraphs, Discuss. Math. Graph Theory 37 (2017), 261-272.

[13] L. Volkmann, Signed total Roman $k$-domination in graphs, J. Combin. Math. Combin. Comput. 105 (2018), 105-116.

[14] L. Volkmann, Weak signed Roman domiation in graphs, Commun. Comb. Optim. 5 (2020), 111-123.

Lutz Volkmann Lehrstuhl II für Mathematik, RWTH Aachen University, 52056 Aachen, Germany

E-mail: volkm@math2.rwth-aachen.de 